\title{
An Assessment of Quality Assurance in HIV and AIDS-Related Services in Chivuna Rural Health Facility of Southern Zambia
}

\author{
Harriet Ntalasha*, Simona J. Simona, Felicitas Moyo, Jairous Miti, Ireen Kabembo, \\ Eliphas Machacha, Mundia Libati, Emily Ng'andu, Aurick Mubita
}

Department of Social Work and Sociology, University of Zambia, Lusaka, Zambia

Email: *harriet.ntalasha@unza.zm

How to cite this paper: Ntalasha, H., Simona, S.J., Moyo, F., Miti, J., Kabembo, I., Machacha, E., Libati, M., Ng'andu, E. and Mubita, A. (2018) An Assessment of Quality Assurance in HIV and AIDS-Related Services in Chivuna Rural Health Facility of Southern Zambia. World Journal of AIDS, 8, 37-52.

https://doi.org/10.4236/wja.2018.81004

Received: March 11, 2018

Accepted: March 26, 2018

Published: March 29, 2018

Copyright $\odot 2018$ by authors and Scientific Research Publishing Inc. This work is licensed under the Creative Commons Attribution International License (CC BY 4.0).

http://creativecommons.org/licenses/by/4.0/

\section{(c) (i) Open Access}

\begin{abstract}
With many countries experiencing high prevalence rates of HIV scaling up ART, it is vital to assess quality assurance in health facilities accredited to provide these HIV and AIDS-related services. Reviewed literature indicates that there are limited studies in Zambia on the capacity of accredited health care facilities to provide effective HIV/AIDS related services. Using data from a large ethnographic qualitative study in a resource poor rural setting in Zambia, this paper assesses quality assurance in health facilities to providing HIV/AIDS services in a remote rural setting. Findings show that although HIV and AIDS related services were available at the remote rural health facility of Chivuna, the services provided did not meet the WHO minimum guidelines/standards on the provision of such services. Therefore, there is need for such facilities to be adequately equipped in all the departments of ART delivery so as to ensure effective delivery of these services and universal access.
\end{abstract}

\section{Keywords}

Zambia, HIV and AIDS, WHO Guidelines, Capacity of Health Facilities

\section{Introduction}

Like many other countries with grappling with relatively high (13\%) prevalence rates of the HIV and AIDS pandemic within the Sub-Saharan Africa, Zambia has scaled up HIV/AIDS-related services. In this regard, the government of the Republic of Zambia has accredited a number of health centers to offer HIV/AIDS-related services so as to maximize accessibility as well as monitor the quality of services offered to the population. Inadequate literature, however, exists on the capacity of 
these facilities to offer HIV/AIDs-related services in line with the key principles underpinning the accreditation framework of health care services for people living with HIV/AIDS [1]. This is the gap in knowledge that this paper attempts to fill.

The World Health Organization (WHO) posits that quality assurance is premised on a structure-process-outcome framework. This means that it has an objective of improving the outcome of a broad spectrum of health care in terms of health, functional ability and the well-being and satisfaction of health care users [1] [2]. The main purpose is to get everyone involved in health care provision to take an active role in supporting quality care, intensifying performance-related problems and taking responsibility for setting in motion the needed changes to improve care.

In the 2004 report of a consultation meeting on the accreditation of health care service facilities, WHO stipulated guidelines for quality assurance in HIV care. The guidelines were not meant to be blueprints of care but were to be adopted according to member countries' capacity and prevailing social environment. In Zambia, the guidelines include issues related to HIV counseling and testing, human resource, physical infrastructure, supply of ARVs drugs and laboratory management [3] [4] and it is against these that this paper assesses the research site.

The guidelines stipulate that a facility offering HIV and AIDS-related-services should have an established policy and follow a written policy on HIV testing. This policy should reflect national laws and WHO guidelines for rapid testing [2]. Additionally, all staff should be familiar with the policy. In terms of counseling, there should be both pre-test and post-test counselling and HIV testing should be conducted with informed consent by individuals trained for the task. The counselling should take place in an environment that ensures privacy. Testing should adhere to $5 \mathrm{Cs}$ of consent, confidentiality, counselling, correct results, and connection to care, treatment and prevention services [3].

In terms of human resource, a facility should have an ART Clinical Officer (CO) permanently located at the ART clinic, two nurses-one for drug dispensary and the other for adherence, 4 community adherence supporters and data entry specialist/health communication officer. All members of staff working in such a facility should be adequately trained and certified to prescribe ART, follow up recipients clinically and also be able to handle HIV and AIDs related issues and conditions that may confront them [2]. This includes both adult and pediatric ART.

Such a facility should have at least six rooms consisting of a screening room, registry room with secure and confidential patient record system with lockable cupboards. In addition to this, there should be a special counselling room, waiting room, information room and dispensing room with stock control cards and air condition to keep the drugs at the right temperature.

Uninterrupted supply of ARVs and ART provision is also one of the stan- 
dards. This was meant to promote adherence to ART [4]. There is also need to support people being treated so as to facilitate their adherence to the prescribed treatment and a written protocol to guide decisions on treatment eligibility.

Laboratory management should include rapid testing, a CD 4 count machine for viral load monitoring, and hematology machine, full serum chemistry analyzer, mycobacterial culture, sputum smear microscopy for TB detection, hemoglobin and HIV antibody test machines [1] [2] [4]. Additionally, the laboratory should meet National Guidelines and tests norms or WHO guidelines. In terms of clinic frequency or opening times, the clinic should be provided at least five days in a week.

In sub-Saharan Africa, a number of studies have been carried out to assess the quality of care and capacity of health facilities accredited to provide HIV-related treatment in resource-limited settings [5] [6] [7] [8]. Although these studies have reported positive out looks in a few health facilities, they have largely shown inadequacies in the health delivery system. The studies for instance indicated that there were inadequate trained personnel in HIV and AIDS related services and ART management [5] [8] inadequate laboratory equipment for ART monitoring, inadequate confidential places for counseling and information system was weak [5] [9]. Others were the inability of all the eligible patients to start ART due to inadequate ARVs and persistent drug stock-outs [6] [7] [8].

In Zambia, reviewed literature indicates that there are no studies done to assess quality assurance among accredited health facilities. The extent to which accredited health facilities are suitable to offer HIV-related services is thus unknown. This article reports findings from a study that was conducted in the rural site so as to profile the characteristics of health facility from a resource constrained country but also a rural one where conditions are expected to be in worst shape.

\section{Methods}

\subsection{Study Area}

The study was carried out in Chivuna, a rural community located approximately $60 \mathrm{~km}$ South-East of Mazabuka town, $35 \mathrm{Km}$ from the Great North Road and about $70 \mathrm{~km}$ South of Lusaka, the capital city of Zambia. The main ethnic group is the Tonga speaking (or $\mathrm{Ba}$-Tonga in plural), a matrilineal and patrilocal group of people. Chivuna has a population of approximately 19,000 scattered in an area covering about 34 square kilometers. The main source of livelihood is subsistence farming, which is seasonal and dependent on the rainfall pattern. Like most rural areas in Zambia, poverty levels are high, averaging more than seven people in ten being poor and having limited access to basic necessities, including food, shelter and health care. While nearly all the health facilities in the area offer VCT, ART services only exist at Chivuna and Mbayamusuma health facilities. The two facilities have a distance of more than $40 \mathrm{~km}$ apart. Chivuna health facility started providing VCT and ART in 2006 and 2008 respectively. 


\subsection{Data Collection and Analysis}

The data presented here was part of a bigger qualitative, ethnographic study conducted over a period of one and half years. This component of the study assessed quality assurance at Chivuna facility being one of the health facilities accredited to provide ART in the District. The facility was visited several times over the study period. In-depth interviews with professional and lay staff, Focus Group Discussions (FGDs) and observations of available resources were conducted. A total 8 professional health care providers, 6 community health workers and 10 ART clients were interviewed. FGDs were conducted with ART clients where there we had 2 from the health facility and the other 2 from the community. Observations were done with respect to staffing levels, available space, essential equipment, drug supplies, laboratory systems and patient-provider interactions/relationships among others. Interviews with health staff were conducted in English while those with community members were done in the local language, Chitonga. Purposive sampling was used to recruit all study participants.

For the purpose of quality control, all the interviews and observations were carried out by the principal investigator. Repeated observations were conducted at the health facility. All interviews were transcribed verbatim and word-processed and the analysis was done using Atlas-ti version 7 using grounded thematic coding, starting with open coding after which all the codes were grouped into similar categories. From these categories, themes were generated.

\subsection{Ethical Approval}

Ethical approval for the study was obtained from the Research Ethics Committee of the school of medicine at the University of Zambia and it was also cleared by the Ministry of Health. For all the individuals that took part in the study, informed consent was administered and participants were made to sign and for those who could read or right thumbprints were used. Before consenting, each participant was asked if they understood the risks and benefits of taking part in the study. Anonymity and confidentiality were assured by way of not disclosing actual identities of participants in the interview tools and subsequent publications.

\section{Results}

The assessment quality assurance addressed questions related to HIV/AIDs services offered and where were they located, when they were administered, who was in charge of the administration and under what conditions. Focus was also on the infrastructure, staffing levels, training of staff who administer ARVs, the availability of essential drugs, reagents and equipment such as CD4 count and liver functioning machine. The results are reported are reported with respect to these parameters:

\subsection{Provision of VCT and ART Services}

Chivuna Rural health facility offers both VCT and ART services. The facility has 
been providing VCT and ART services since 2006 and 2008 respectively. These services were provided three times per week on Mondays and Thursdays (with Wednesdays restricted for pediatric ART). ART was not integrated into general health services but was separated from the rest of the services. This practice was to some extent viewed with mixed feelings by some clients. While some felt that it exposed them to finger pointing and stigmatization, the majority seemed to hold a few that it was better as it gave them an opportunity for interaction and to share experiences among themselves as fellow positive people. Such experiences were viewed as facilitating factors for adherence. There was a TB and youth-friendly corner though at the time of the study the youth friendly corner was not yet in operation. This seemed to create a lot of challenges for youths who were visibly missing from the facility.

The health facility was also involved in provision of mobile services which included VCT and community-based sensitization. This was done in order to provide services to many remote communities, which were still affected by long distances to health centers. These mobile services were provided with the support of NGOs such as Churches Association of Zambia (CHAZ) and Total Control of the Epidemic (TCE) a Danish funded NGO. The main services offered under the mobile services were Prevention of Mother to Child Transmission (PMTCT), under five clinic, antenatal clinics, community sensitization, family planning and VCT. CHAZ had also bought the facility a $4 \times 4$ vehicle specifically for mobile VCT. However, provision of these community-based activities was at times not consistent due to inadequate and erratic funding as well as shortage of staff. For instance, although the health facility had more than two vehicles, with one vehicle specifically for the ART clinic, the whole facility only had one driver for all the duties at the center. At times fuel was not available. These limitations were ably presented by one of the male health care providers working at the ART clinic when he elucidated:

The available number of staff is at times inadequate to go out in the communities to carry out outreach programs such as community sensitization and mobile ART services because we now have two clinics to run yet the number of staff has remained the same. The same members of staff who work at the ART clinic also conduct general health services. We are also supposed to have two drivers but we only have one at the moment, so it is quite difficult for us even if we have a vehicle specifically meant for the ART clinic. Sometimes you find that you arrange to go out for sensitization or to do mobile ART services, but in the meanwhile there are other operations somewhere that need a driver, or maybe there is an emergency. This means that we have to abandon whatever other activities we had planned.

The hilly and rugged terrain of the area and generally poor state of the road network made some communities inaccessible particularly during the rainy season. For TCE, this was confounded by the use of bicycles as the main mode of transport. However, even when a $4 \times 4$ vehicle was available; some areas were 
still not accessible by such kind of vehicle. In reference to poor road network and other limitations, another health worker noted:

However these mobile services are usually hampered due to lack of financial resources for fuel and manpower. With such constraints, it is usually difficult to say or plan the number of visits we are going to do per month, it is not frequent, so we only do it as and when the resources are there. Then we have the poor road network, in some places, roads do not exist, we have a 4 $\times 4$ but we can't still reach certain places.

As part of community services, the facility also provided sensitization on HIV and AIDS. Being run by the Roman Catholic Church, the facility did not distribute condoms. However, community sensitization activities were hampered by the same limitations affecting mobile VCT, of inadequate staffing, poor road network and inadequate funding.

For follow ups, the facility depended on community treatment supporters. The study discovered that the mechanism had a lot of challenges particularly, for remote communities due to lack of transport. However, there was optimism among staff that the mechanism would improve as the facility was in the process of acquiring bicycles for treatment supporters covering hard-to-reach areas. The poor follow up mechanism was also attributed to what one care provider referred to as, "Allowance syndrome" making some health staff and community health workers not being interested in any extra work without an allowance.

\subsection{VCT and ART Physical Infrastructure}

The building at the hospital which housed ART clinic was specifically built for that purpose. However, the space was clearly inadequate, leading to congestion. The building had three rooms consisting of a waiting room, registry, a consultation room and a tiny corridor with a sitting capacity of about ten people. The waiting room, which was also the group counselling room, could only accommodate about twenty people at a time. According to health care providers this made group counselling, which was supposed to be conducted on each ART day difficult. Yet clients saw group counselling sessions as very important for adherence. The study discovered that, though very critical, special private counselling rooms were not available. The screening and dispensing of drugs were done in the same room while the registry was also the counselling room. Clients waiting to see the clinical officer and those collecting files waited in the small corridor which was very close to the counselling room. It was observed that due to the corridor being too small, some clients sat right in front of the room where counselling was taking place. This required both the counsellor and the client to speak in low tones knowing that there were people sitting right in front of the room. The data/health communication room was also lacking.

These inadequacies in terms of infrastructure had implications on quality of services provide by the facility. For instance, inadequate space had negative implications on the quality of counselling and other services offered. This is clear 
from the concerns raised by both key informants and community members who viewed it as compromising privacy and confidentiality required for counselling. These concerns can be illustrated by the following quotes:

We also need a bigger building so as to accommodate all these services that we are providing. For counselling we need privacy so that patients can be free and comfortable. At the moment this is lacking and as a result clients are limited as to how much they can share with the counsellor because instead of having only 2 people in a room there are usually more than two people in the same room because registry is also the counselling room. So even when they happen to have questions about their treatment and condition, some may not say anything (Male health care provider).

The rooms are not enough, you find in the room there are many of you, maybe more than three yet you are supposed to be only two of you with the counsellor, there is no privacy at all. Most of the time as you are talking to the counsellor there is another nurse giving out cards and people keep coming in and going out of the same room and you get distracted.(male ARV user, male clinic based FGD amid agreement and support from other discussants).

Observations and discussions with health care providers revealed that clients' records were kept on open shelves yet the WHO guidelines recommend that such records should be kept in lockable storage for confidentiality purposes.

\subsection{VCT and ART Staff}

The staff from the main facility worked at the ART clinic on rotational basis. Among the reasons given for this were the inadequate staffing and need for all staff to have experience in providing ART services. Another reason given was to enable all staff working at the facility to have a share in the allowances which were provided by CHAZ for the ART clinic. Inadequate staffing was also cited as partly the reason why the clinic was held only twice in a week.

Consultations with health providers revealed that, on each ART day, there were supposed to be a clinical officer, registered nurse, a pharmacist, a nurse for bleeding, three four counsellors for adherence and an information officer. However, this was not always the case. For instance, at times only one to two staff were found at the facility to conduct all the procedures. Additionally, the facility was supposed to have two clinical officers (COs) but only had one to attend to the Out Patient Department (OPD), In-Patient Department (IPD) and ART clinic. As a result, the clinical officer had to first do the rounds in the wards and attend to any emergencies at the OPD before going to the ART clinic.

Generally, most of the members of staff at the facility complained of being over-worked since the introduction of VCT and ART services. It was pointed out that the new services were being offered in addition to the other duties yet the number of members staff remained exactly the same. This was a great constraint and affected service delivery to people intending to access HIV and AIDS 
related services in many ways. Firstly, it was observed that because of inadequate staffing, people waited for a long time before being attended to. The fact that the whole facility only had one clinical officer and he was required to start with general health service delivery and was only able to go to the ART clinic after 10:00 hours yet some clients came as early as 5:00 hrs. Secondly, it was discovered that counselling sessions were much shorter than they were supposed to be. For instance, one of the health care providers informed the researcher that these counselling sessions were supposed to last not less than 20 minutes but because the counsellors were not enough, counselling time was much shorter. These deficiencies imply that the quality of services delivered was greatly compromised. Views from one of the health care providers are illustrative of this situation when she narrated that:

The ART clinic was introduced without any changes in the number of staff. This has led to increased work load for the few staff available. For instance, the full establishment for the health facility even without the ART clinic is two clinical officers but we only have one. So on each ART day, the only clinical officer has to first do the rounds in the wards and attend to any emergencies at the OPD before going to the ART clinic. Not having enough staff is also partly the reason why the ART clinic is limited to only two days in a week.

Similar sentiments were expressed by several community members as illustrated by the following remarks:

The attitude of most health care providers is just okay but the problem is that the doctor (referring to the clinical officer) is just alone and patients are just too many and no one to help. Some people can wake up around 4:00 hours and they are here by $600 \mathrm{hrs}$. and they would be number 3 or so but the doctor only comes around $900 \mathrm{hrs}$. The doctor has to work at OPD and wards and after she has finished, that is when she attends to patients at ART. Even if you were the first one to arrive, you will go home around 13:00 hours. The workload is just too heavy for one person, if the government could at least send one or two more doctors so that they can assist each other (ART user a mid agreement and support from other discussants, female community based FGD).

According to the health care providers interviewed, their delivery of other services such as counseling was equally affected. These concerns can be elucidated by the view of one of the male health care providers when he stated that:

Even counseling if it has to be adequately done we need more counsellors considering the numbers of patients that we deal with. We are supposed to be with one patient for at least not less than 20 minutes but because sometimes there would be a lot of people to be seen and just a few of you to at- 
tend to them, this becomes very difficult. And also because many of them cover long distances to reach the clinic, you try to squeeze in as many as possible to ensure that all are attended to and by the end of the day you are completely exhausted. This also affects the way some health staff behave towards patients because they are human beings and they get tired.

\subsection{Training in HIV and AIDS-Related Services}

Only the clinical officer (CO) and the ART Coordinator were found to have official training in ART services. These services consisted of adult ART management, pediatrics and VCT diagnostic and counselling. It was revealed that nine health care providers were trained in counselling with only one nurse having been oriented to carry out all the requirements for dispensing of ART yet they were all expected to carry out HIV and AIDS-related services.

The training was provided by CHAZ. However, the study discovered that even when a nurse had been fully oriented in all the areas of ART services, she or he could not initiate treatment because this was not allowed by the national guidelines if neither the clinical officer nor doctor were around.

All the community cadres who included peer educators, HIV medics, treatment and adherence supporters, were found to have only done some basic orientation to ART services for a week. In the actual fact, nearly all the community cadres complained of the training received not being adequate. Most of them stated that the training they covered in one week should have been done in three months implying that most of the training material was left out. Thus, expressing concerns about training needs, a male lay counselor remarked:

We definitely need further training in ART delivery because so far we have just been oriented for one week, under normal circumstances, the course we did should have been done in two months but it was compressed and so we covered it in one week. Though we were trained in both counselling and palliative care, this is still not enough. We need more training in all areas of ART.

In a related interview, a female health care provider also noted:

All members of staff need more training so that they can be competent in ART delivery, at the moment only the $\mathrm{CO}$ and the ART coordinator have had official training in ART services, the rest of the staff have only undergone orientation for one week and this is not enough to enable them adequately handle all the issues that relate to provision of ART services.

It was also revealed that at times health care providers who were adequately trained in ART delivery were not always working at the ART clinic as staff worked at the clinic on rotational basis. In essence, this compounded the inadequate training of staff in ART delivery. Gaining experience from working in the ART clinic would have been a cushion for inadequate training. For instance, it 
was found that the only nurse who had been oriented in all the areas of ART delivery and once coordinator for the ART clinic was at the time of the study, no longer working at the ART clinic but at the OPD. This did not only disrupt continuity in experience but was also a clear misplacement of training and experience yet both were very essential for a specialized field like ART delivery. According to some of the users, this was viewed as compromising confidentiality, which can also be a limiting factor to those people who want to access and utilize available services.

\subsection{VCT and ART Equipment}

In terms of laboratory management the only equipment that the facility had was as sputum smear microscope for latent TB diagnosis. Reagents were also always available through the involvement of both the government and CHAZ in the distribution of these items. At times Total Control of the Epidemic (TCE), a cooperating partner in VCT, also provided testing reagents. This made basic testing such as rapid testing always possible. However, major equipment such as CD4 count machine for viral load monitoring, sputum test machine, hematology and, hemoglobin machines, full serum chemistry analyzer and antibody test machines were all lacking.

Samples for liver and kidney functioning were being sent to the District Hospital because the facility lacked a chemistry analyzer and CD4 count machine. This was done two times a month. There was much concern by health care providers over the lack of these basic but essential testing machines because this required that all samples delivered to Mazabuka District Hospital located more than $60 \mathrm{~km}$ away. This was a big challenge particularly during the rainy season as the road connecting the two health facilities was at times impassable. At times the health facility also experienced logistical problems. All this meant re-taking blood samples, a situation that sometimes raised suspicions and resistance from some of the members of the community who suspected some health care providers of being involved in Satanism, thereby using their blood for rituals. This resulted into reluctance on the part of some potential users and dissuaded some of those believing such allegations from HIV testing, and consequently limiting the access to ART.

Lack of testing equipment was reported to lead to delays in getting test results and consequently caused delays in linking positive clients to care and initiating treatment for eligible clients as a confirmed HIV test result was required before treatment could be initiated. Many study participants from both the health facility and the community narrated how lack of equipment delayed people to get their test results, be linked to care and be initiated on ART. Some of these experiences are expressed in the quotes below:

The frequency of transporting blood samples increases depending on the number of people queuing up for testing in a particular month. The problem is that sometimes the machine breaks down on the day the samples are 
taken due to too much pressure because we are not the only health facility that takes samples there. When this happens, blood samples have to be retaken because we cannot keep them beyond $24 \mathrm{hrs}$. before testing. This leads to delay in initiating patients on medication because there is a requirement that results are known before a client can be put on drugs (Male health care provide).

Some testing machines should be acquired because that is one thing that delays people to start medication, some die while waiting for test results because our blood has to be taken to Mazabuka and then sometimes we are told that there was need for fresh blood and tests to be repeated meanwhile the patient keeps waiting for results without any medication even when one could be very ill (middle aged female ARV user, clinic based FGD).

If only the samples could be tested here [at Chivuna health facility] it would have been better but now patients have to wait for samples to be taken to Mazabuka and then results to come before somebody can be given the drugs, this causes a lot of delay, some of our colleagues have died in the process of waiting (male on ART for 3 years, clinic based IDI).

To some extent the delays and the re-taking of blood samples lead to mistrust and suspicions of some health care providers. It was also suspected that repeated taking of blood samples was to enable some health care providers use the collected blood for satanic rituals. Further, it was revealed that some eligible clients were in the process of waiting for test results lost. One ART client stated that:

That happens a lot [referring to retaking of blood samples], like for me, they had collected blood more than two times from me. Because of drawing blood from you on a daily basis in the end, they can even drain the last of drop of blood from you, sometimes you start thinking of possibility of other things happening, we do not know what happens maybe our blood is being sold (Female ARV user, mixed clinic based FGD, amid agreement from other participants).

Such concerns were confirmed by some health care providers as one of them stated that:

Yes there is some talking about that [repeated blood taking]. Some clients have even approached me jokingly asking about where we take their blood and whether we sell it. They have heard about Satanism yes and so they ask such questions. This is made worse by the re-taking of blood samples we have talked about. And then results do delay sometimes because of not having our own machines here. Before our CD4 count machine was taken to Chikankata Hospital things were much better. We have lost some patients in this manner (male health care provider.)

Some health staff expressed how frustrated they felt at times when they were unable to initiate treatment due to delayed test results even when some clients 
would be showing signs of deteriorating health status. It was further reported that in the beginning, a CD4 count machine was allocated to the facility by CHAZ. This equipment had since been transferred to another much bigger health facility located about more than 50 kilometres away. In addition to this, the facility also lacked many other equipment required as minimum standard for a facility offering HIV and AIDS-related services.

\subsection{Supply of HIV and AIDS Drugs}

All the drugs required for the administration of ART were provided by both $\mathrm{CHAZ}$ and government through medical stores. The facility had never experienced any drug shortages and had in stock both first line and second line ARVs. The readily and consistent availability of ARV drugs was an important predictor for drug compliance, an assertion verified by all sources, including physical checks on all ART days. The readily availability of ARVs encouraged people to cover the long distances to the health facility and was hence a facilitator for adherence. Antibiotics for prophylaxis were also always available. This finding was unique to the study area because at the time of the study there were protests in other parts of the country due to shortages of HIV and AIDS-related drugs, particularly ARVs. There was Post-Exposure Prophylaxes (PEP) only for members of staff. However, at the time of the study incidences requiring these services such as accidental needle pricking of staff had never occurred at the facility.

\section{Discussion}

The study sought out to assess quality assurance in HIV/AIDs-related services in Chivuna Health facility and generally, the findings indicate that HIV/AIDS services were available at the facility. However, as observed before by other qualitative studies in the region [7] [8], the present study identified a number of gaps when compared with the WHO minimum standard guidelines in provision of VCT and ART services. The results from our study do confirm that these inadequacies affected the effective operations of the facility. The gaps did exist in nearly all the key departments of VCT and ART delivery. In laboratory management for example, the CD4 count, the hematology machine and chemistry analyzer were lacking. As observed by [8], this inevitably contributes to delays in people getting their results and consequently delays in those found HIV positive in accessing the care and treatment they require. Inadequate laboratory equipment also affected ART monitoring. This has also been observed by other authors [5] [6].

Physical infrastructure was also observed to be below the WHO standards. Instead of having at least six rooms for counselling, screening, record keeping, counselling, waiting room, dispensing and data room as per minimum requirements, our results reveal that Chivuna health facility only had three rooms. This led to combining of some of the services in a single room. This also meant that 
some of the services provided were not as effective as they are expected to be. For instance, because of lack of specific counselling rooms, the quality of counselling was compromised due to confidentiality concerns. The situation also has potential to prevent clients from seeing seeking counselling at the facility.

Study findings also reveal, a finding not unique to this study but common in many earlier studies also [10] [11] [12] [13] the facility experienced staff shortages. Our findings show that staff shortages impacted negatively on counselling, waiting and opening times. For instance, because of low staffing levels, our findings show that the length of counseling sessions was reduced. In the same vein, because of inadequate staffing, particularly in relation to the fact that there was only one clinical officer to carry out all the services at the facility, people were subjected to longer waiting times. And as earlier reported [14] [15] [16], inadequate staffing leads to burn out among health care providers because they end up being over worked, a situation that compromise the quality of care delivered. In Chivuna, shortage of staff was also the reason given for providing ART only three times in a week.

Training in HIV and AIDS-related services was also inadequate. The lack of training in a way contributed to the lack of confidentiality among some of the health staff, which equally emerged as a barrier to people entering and being retained in the HIV continuum of care. Like inadequate staffing alluded to above, inadequate training in ART delivery negatively impact on the quality provided. In all their studies from Botswana, Uganda and Tanzania, [15], cite the importance of having well trained health care staff in ensuring provision of quality HIV and AIDS-related counselling as a way of enhancing access to HIV and AIDS-related services.

Lack of trained staff in ART is both a risk to staff and clients and may compound the problems. When clients become aware that staff are not adequately trained, they may lose confidence in the facility and possibly resort to other means such as traditional medicines which are largely neither scientifically tested nor approved. Lack of training among ART staff and its negative effects has been reported also by other qualitative studies from other countries within the region [9] [14] [17] and from Zambia [18] [19] [20]. In our study area, lack of training was compounded by lack of continuity in service delivery by health staff in that they worked at the ART clinic on a rotational basis.

Generally, as observed above all these gaps had negative implications on the effectiveness of the services delivered. Earlier studies within the region [8] [10] [17] [21] all observe inadequacies in many health institution accredited to provide HIV- and AIDS-related services. The inadequacies in the health delivery system identified above affected the effectiveness in the delivery of HIV and AIDS-related services.

It should be mentioned nonetheless, that the fact that Chivuna health facility never run out of ARV drugs, both first line and second line drugs, was an important predictor for drug compliance because it encouraged people living with 
HIV to cover inconceivably long distances to the health facility knowing that they will find them each time they went there. As stipulated by the WHO guidelines, uninterrupted provision of ARVs was critical so as to avoid treatment interruptions if drug resistance is to be prevented and also make ART effective. This was an interesting finding considering that a number of previous studies have reported of drug shortages or irregular medical supplies due to weak procurement procedures as being critical barrier to ART access and adherence [8] [14] [19] [22] [23]. Additionally, Chivuna health facility had sputum machine and always had reagents for various tests.

\section{Conclusion}

The findings from this study have shown that while HIV/AIDs-related services were available in rural settings like Chivuna, a number of gaps were recorded. The findings indicate that a few services, such as uninterrupted supply of ARVs and availability of reagents were in line with WHO guidelines. However, for the majority of the services were below the WHO minimum standard guidelines for the provision of such services. These inadequacies in the health delivery system contributed to poor service delivery. Therefore, for HIV and AIDS-related services to be effective and also ensure universal access to these lifesaving services, there is need for government and cooperating partners to address these inadequacies so as to optimize access to care for people living with HIV. This is important because provision of health care in itself is not enough with assuring the quality of care provided and hence the need to address it.

\section{Acknowledgements}

This study was part of the ART access project and it was by funded by The Swiss National Science Foundation (SNF) through the Swiss Tropical and Public Health Institute. We acknowledge this funding. We also acknowledge the support from our host institution, the University of Zambia for the space and time to work on this paper. The views expressed in this paper are entirely those of the authors.

\section{References}

[1] WHO (2004) Standards for Quality HIV Care: A Tool for Quality Assessment, Improvement and Accreditation.

http://www.who.int/hiv/pub/prev_care/en/standardsquality.pdf

[2] WHO/UNAIDS Guidelines. http://apps.who.int/iris/bitstream/10665/85326/1/9789241505734_eng.pdf

[3] Zambia National Guidelines for HIV Testing and Counseling (2006) http://hivstar.lshtm.ac.uk/files/2016/05/Zambia-National-Guidelines-for-HIV-Cou nsellig-and-Testing2006.pdf

[4] Zambia Consolidated Guidelines for Treatment and Prevention of HIV Infection (2013)

http://www.northriseuniversity.com/wp-content/uploads/2017/07/Zambia-Consoli 
dated-Guidelines-for-Treatment-and-Prevention-of-HIV-Infection-.pdf

[5] Mapunjo, S. and Urassa, D.P. (2007) Quality Standards in Provision of Facility Based HIV Care and Treatment: A Case Study from Dar es Salaam Region, Tanzania. East African Journal of Public Health, 4, 12-18.

[6] Somi, G., et al. (2009) Three Years of HIV and AIDS Care and Treatment Services in Tanzania: Achievements and Challenges. Tanzania Journal of Health Research, 11, 136-143. https://doi.org/10.4314/thrb.v11i3.47700

[7] Mashauri, F.M., Siza, J.E., Temu, M.M., Mngara, J.T., Kishamawe, C. and Changalucha, J.M. (2012) Assessment of Quality Assurance in HIV Testing in Health Facilities in Lake Victoria Zone, Tanzania. Tanzania Journal of Health Research, 9, 110-114.

[8] Olaleye, O.O., Adeokun, A.L., Oronsaye, F., Ladipo, O.A. and Delano, G.E. (2006) Assessing the Level of Preparedness of Private Health Providers for Clinical Management of HIV/AIDS Epidemic in Nassarawa State, Nigeria. African Journal of Reproductive Health, 10, 90-97. https://doi.org/10.2307/30032475

[9] Roura, M., Busza, J., Wringe, A., Mbata, D., Urassa, M. and Zaba, B. (2009) Barriers to Sustaining Antiretroviral Treatment in Kisesa, Tanzania: A Follow-Up Study to Understand Attrition from the Antiretroviral Program. AIDS Patient Care and STDS, 23, 203-209. https://doi.org/10.1089/apc.2008.0129

[10] Layer, E.H., Kennedy, C.E., Beckham, S.W., Mbwambo, J.K., Likindikoki, S., et al. (2014) Multi-Level Factors Affecting Entry into and Engagement in the HIV Continuum of Care in Iringa, Tanzania. PLOS ONE, 9, e104961.

https://doi.org/10.1371/journal.pone.0104961

[11] Mabunda, G. (2006) Voluntary HIV Counselling and Testing: Knowledge and Practices in Rural South African Village. Journal of Transcultural Nursing, 17, 23-29. https://doi.org/10.1177/1043659605281978

[12] Vermeulen, J.H. (2011) Identifying Structural Barriers to Adherence. Thesis Presented in Fulfilment of the Requirement for the Degree of Master of ART in (Psychology) at Stellenbosch University.

[13] Mshana, H.G., Wamoyi, J., Busza, J., Zaba, B., Changalucha, J., Kaluvya, S. and Urassa, M. (2006) Barriers to Accessing Antiretroviral Therapy in Kisesa, Tanzania: A Qualitative Study of Early Retroviral Referrals to the National Programme. AIDS Patient Care and STDs, 20, 649-656. https://doi.org/10.1089/apc.2006.20.649

[14] Schouten, E.J., Jahn, A., Ben-Smith, A., Makombe, S.D., Harries, A.D. and Chambwandera, F. (2011) ART Drug Supply Challenges in the Era of Scalling up ART in Malawi. Journal of the International AIDS Society, 1, S4.

[15] Hardon, A.P., Akurut, D., Comoro, C., Ekezie, C., Irunde, H. F., Gerrits, T., Kglatwane, J., Kinsman, J., Kwasa, R., Maridadi, J., Moroka, T.M., Moyo, S., Nakiyemba, A., Nsimba, S., Ogenyi, R., Oyabba, T., Temu, F. and Laing, R. (2007) Hunger, Waiting Times and Transport Costs: Time to Confront Challenges to ART Adherence in Africa. AIDS Care, 19, 658-665.

https://doi.org/10.1080/09540120701244943

[16] Grant, E., Logre, D., Gorman, D. and Murray, S.A. (2008) Factors Facilitating and Challenging Access and Adherence to Antiretroviral Therapy in a Township in the Zambian Copperbelt Province: A Qualitative Study. AIDS Care, 20, 1155-1159. https://doi.org/10.1080/09540120701854634

[17] Libamba, E., Makombe, S.D. and Harries, A.D. (2007) Malawi Contribution to 3 by 5. Achievements and Challenges. Bulletin of the World Health Organization, 85, 156-160. 
[18] Samuels, G., Simbaya, G.J. and Ndubani, P. (2008) Social Aspects of Free AIDS Medicine (ARVs). Access in Public Health Setting in Tanzania and Zambia, Zambia Country Study.

https://www.odi.org/projects/social-and-economics-costs-free-aids-medicines-arvspublic-health-care-settings-tanzania

[19] Sanjobo, N., Frich, J.C. and Fretheim, A. (2008) Barriers and Facilitator to Patient Adherence to Antiretroviral Treatment in Zambia: A Qualitative Study. Journal of Social Aspects of HIVI AIDS, 5, 136-143.

[20] Harries, A.D., Makombe, S.D., Libamaba, E. and Some, E. (2007) Ensuring Uninterrupted Supplies of ART. Bulletin of the World Health Organization, 85, 152-155. https://doi.org/10.2471/BLT.06.032060

[21] Muula, A. (2004) Ethical and Programmatic Challenges in ART Scaling up in Malawi; Challenges in Meeting the WHO, Treating 3 Million by 2005. Croatian Medical Journal, 45, 415-421.

[22] Levy, J. (2009) Women's Expectations of Treatment and Care after an Antenatal HIV Diagnosis in Lilongwe, Malawi. Reproductive Health Matters, 17, 152-161. https://doi.org/10.1016/S0968-8080(09)33436-9

[23] Medley, M. and Kennedy, C.E. (2010) Provider Challenges in Implementing Antenatal Provider Initiated HIV Testing and Counselling Programs in Uganda. AIDS Education and Prevention, 22, 87-99. https://doi.org/10.1521/aeap.2010.22.2.87 